\title{
Agôn
}

Revue des arts de la scène

Critiques | Saison 2015-2016

\section{La Dictature du cool, mise en scène Marco Layera}

Les limites du « bobo»

\section{Caroline Châtelet}

\section{Q OpenEdition}

Journals

Édition électronique

URL : http://journals.openedition.org/agon/3972

DOI : 10.4000/agon.3972

ISSN : 1961-8581

Éditeur

Association Agôn

Référence électronique

Caroline Châtelet, "La Dictature du cool, mise en scène Marco Layera », Agôn [En ligne], Critiques, mis en ligne le 20 août 2016, consulté le 23 septembre 2020. URL : http://journals.openedition.org/agon/ 3972 ; DOl : https://doi.org/10.4000/agon.3972

Ce document a été généré automatiquement le 23 septembre 2020

Association Agôn et les auteurs des articles 


\title{
La Dictature du cool, mise en scène Marco Layera
}

Les limites du «bobo»

\author{
Caroline Châtelet
}

\section{RÉFÉRENCE}

La Dictature du cool mise en scène Marco Layera, compagnie la Re-sentida

Festival IN d'Avignon, du 18 au 24 juillet 2016 - Gymnase du Lycée Aubanel

1 Voir un (autre) spectacle : programmé (lui aussi) dans le cadre du IN d'Avignon; dont on a entendu le pire comme le meilleur; interprété par la Re-Sentida, compagnie chilienne qui a déjà défrayé la chronique lors de sa première venue au festival en 2014. Se (re)dire que ce n'est qu'une des caractéristiques de cette caisse de résonance festivalière, où les polémiques s'exacerbent à tel point qu'on lancerait bien à la cantonade "on se détend, les youngs, ce n'est que du théâtre». Être tout de même sacrément curieuse, eu égard au pugilat à l'article signé José Pons dans les Inrocks (« Du pus sur les planches: les nouveaux réactionnaires s'emparent d'Avignon $\left.{ }^{1} »\right)$. Découvrir, enfin, La Dictature du cool, qui raconte, lors d'un 1er mai au Chili, la célébration par une poignée d'ami.e.s de l'accession de l'un d'entre eux au poste de Ministre de la culture. Ce dernier dévoilera au cours de la soirée à ses proches dont il exècre désormais le cynisme, l'hypocrisie et le carriérisme, la liste de ses futurs collaborateurs : un militant syndical, un paysan, un défenseur des droits des Mapuches, minorité indienne ostracisée par le pouvoir chilien, etc. Pendant que dans les rues, les manifestations du 1er mai dégénèrent, dans le huis-clos de l'appartement, tous tentent de faire revenir leur ami à la raison, espérant un rôle au sein du portefeuille. Au fil du spectacle et de ses excès - la forme étant à l'image de ce qu'elle entend dénoncer -, le dégoût puissant, comme l'adhésion que cette création a provoquée, interroge. Au-delà de ces réactions éruptives, La Dictature du cool se donne en effet comme une satire, formellement assez bien ficelée, dont les deux points d'achoppement seraient : l'écart entre son ambition 
affichée et la réalité de son discours; son postulat erroné en ce qu'il appréhende les «bobos » comme un groupe social homogène. Alors, certes, ces deux (bons gros) problèmes de fond neutralisent toute portée critique. Mais une fois admis ces écueils, la création se donne comme ce qu'elle est: une forme satirique, donc, utilisant tous les artifices de son genre: exagération, caricature, parodie. Sur le plateau, le metteur en scène Marco Layera installe une scénographie juxtaposant des espaces aux fonctions précises. Tandis qu'à l'avant-scène se trouve un salon, l'arrière-scène est découpé en trois zones. Côté jardin, les loges, où lorsque le spectacle débute les comédiens se préparent sous l'œil de caméras. Tandis qu'un écran situé à l'avant-scène relaie ces images, la voix off du Ministre de la Culture les commente, détaillant les métiers (employé dans une O.N.G., technicien dans une structure culturelle, comédien, performer, etc.), critiquant les travers de chacun. Une première scène éloquente qui, en convoquant les codes de la télé-réalité, brouille la distance entre personnages et acteurs et souligne que chacun ici joue, sait qu'il est en permanence en représentation et soumis au regard d'autrui. Puis, on trouve une galerie d'art où des tags côtoient des néons, clin d'œil à la récupération par le marché de l'art de toute création contestataire et marginale. Un lieu dans lequel la performeuse tentera, dans une confidence à la facticité appuyée, de faire changer le Ministre d'avis. Enfin, côté cour, des escaliers et des couloirs composent un espace où les personnages, toujours selon les codes de la télé-réalité, s'épuisent, courant, pleurant, hurlant face caméra. Dans un jeu volontairement outré, la bande de quarantenaires ne cesse d'ailleurs de brailler, taper de la cocaïne, boire, danser ou chanter. Cette mise en tension et cette hystérie perpétuelles, soutenues par le recours à la vidéo et à la musique (techno ou électro et poussée à son maximum) contrastent volontairement avec les discours et les appels à une vie saine, proche de la terre, empreinte de spiritualité. En repoussoir de la bande bien peu aimable se trouverait le Ministre. Un piètre contre-modèle, cynique comme les autres - avec le grotesque de son cabinet à la pseudo «diversité »-, qui prend un malin plaisir à pousser chacun dans le mensonge, la dissimulation. Aucun n'est à sauver, et même la recherche de l'authenticité s'avère façonnée par l'entertainment et les médias. Le résultat est une forme épuisante, qui en convoquant volontairement tous les artifices de ce qu'elle entend dénoncer, soumet les spectateurs à un univers en tension, où la distance critique s'avère difficile. Outre ces limites liées au choix formel, d'autres, plus problématiques, apparaissent. D'abord, Marco Layera explique à longueur d'interview vouloir critiquer les «bobos", actifs urbains au pouvoir d'achat conséquent, parfaitement adaptés à un système néolibéral qu'ils ne cessent paradoxalement de critiquer et que le metteur en scène définit comme un groupe social ${ }^{2}$. Sauf que l'usage de ce terme, théorisé par le journaliste américain David Brooks en $2000^{3}$, ne recouvre aucune réalité sociale concrète, ne se fonde sur aucun travail scientifique et évacue l'idée de luttes de classe ${ }^{4}$. Ensuite, comme l'a pertinemment relevé Eric Sénabre, romancier jeunesse, dans une tribune parue dans Libération ${ }^{5}$, «Le "bobo" est devenu l'homme à abattre des politiciens de droite. Sa seule présence en un lieu, dans un rassemblement, un mouvement, suffit à y porter le discrédit. » Le mot n'est jamais utilisé à titre mélioratif, valorisant ou même bienveillant. Il constitue un moyen de disqualification à peu de frais d'une personne, d'un groupe et son usage se fait souvent avec mépris ou condescendance, quand il ne s'agit pas carrément de haine. Le «bobo » agrège à lui seul - comme par hasard - les pires tares de la société et soulage au passage chacun de son auto-critique. Pire, comme le rappelle à la journaliste Marion Rousset la sociologue Sylvie Tissot ${ }^{6}$, "C'est un ennemi facile. Sa caricature permet à la droite et à une 
fraction du Parti socialiste de délégitimer des valeurs de gauche : le combat pour les droits humains, contre les inégalités et les discriminations, pour le mariage pour tous ou le droit de vote des étrangers. » Du populisme d'un terme et de ses usages.

\section{La Dictature du cool - mise en scène Marco Layera}

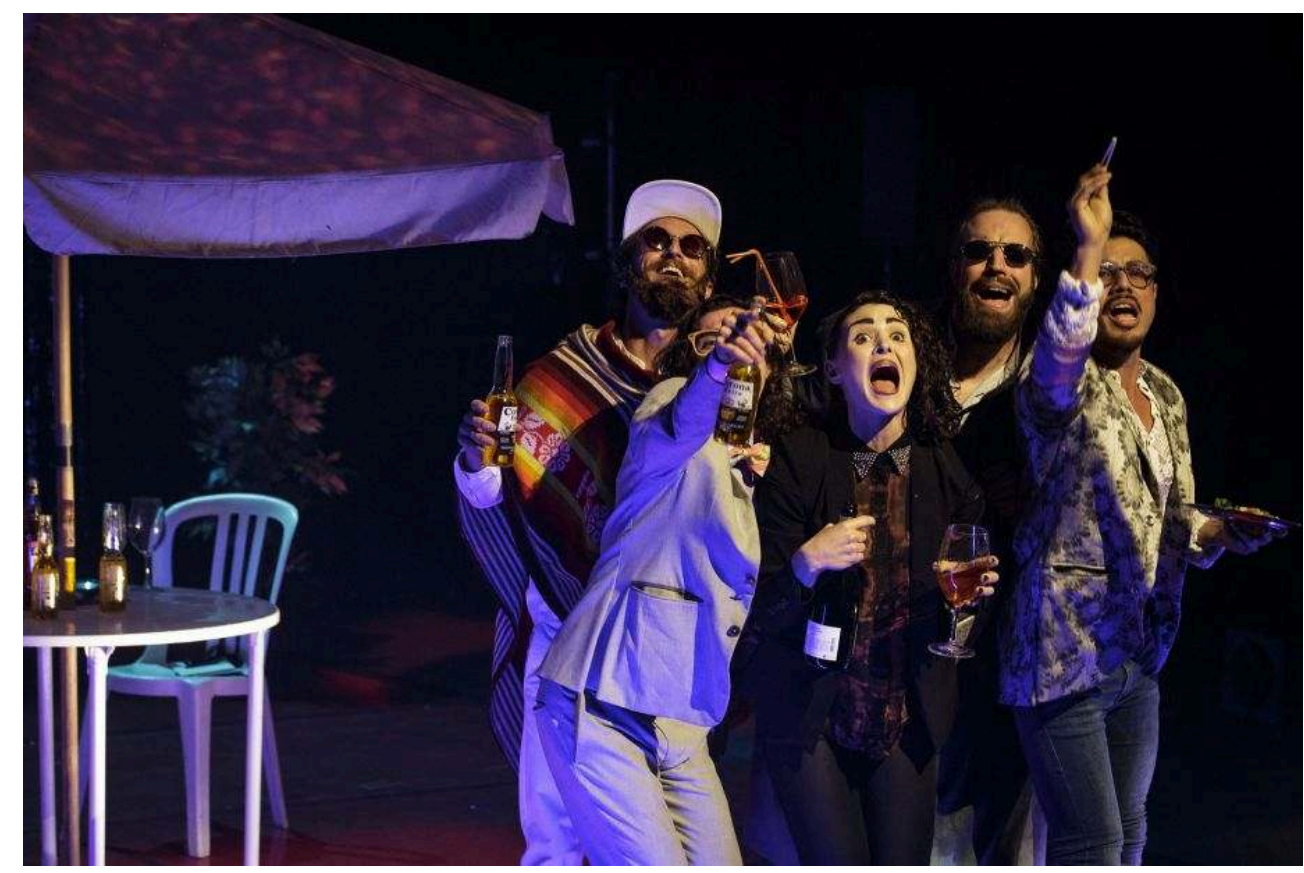

CHRISTOPHE RAYNAUD DE LAgE

\section{NOTES}

1. Voir : http://www.lesinrocks.com/2016/07/21/scenes/pus-planches-nouveauxreactionnaires-semparent-davignon-11854993/

2. Ce faisant, l'équipe chilienne inclut le public dans cette critique - une fausse représentante du gouvernement chilien interpellant les spectateurs avant le début de la représentation par des questions portant sur ses modes de consommation.

3. David Brooks, Les Bobos, "Les bourgeois bohèmes", trad. par M. Thirioux et A. Nabet, Paris, Florent Massot, coll. Le livre de poche, 2000, $314 \mathrm{p}$.

4. À ce sujet, lire l'article de la sociologue Anne Clerval, https://cybergeo.revues.org/766

5. http://www.liberation.fr/debats/2016/04/25/et-si-on-fichait-la-paix-aux-bobos_1448566

6. http://www.telerama.fr/idees/comment-le-bobo-est-devenu-le-coupable-ideal,105236.php 